\title{
Very Late-Onset Friedreich Ataxia with Laryngeal Dystonia
}

\author{
Silvia Rota ${ }^{a} \quad E$ eleonora Marchina ${ }^{b} \quad$ Alice Todeschini $^{a} \quad$ Lorenzo Nanetti $^{c}$ \\ Fabrizio Rinaldi ${ }^{a} \quad$ Alessandra Vanotti ${ }^{c}$ Caterina Mariotti ${ }^{c}$ \\ Alessandro Padovani ${ }^{a}$ Massimiliano Filosto ${ }^{a}$ \\ ${ }^{a}$ Section for Neuromuscular Diseases and Neuropathies, Unit of Clinical Neurology, \\ University Hospital 'Spedali Civili', 'Unit of Biology and Genetics, Department of \\ Molecular and Translational Medicine, University of Brescia, Brescia, and ' $U$ nit of Genetics \\ of Neurodegenerative and Metabolic Diseases, Fondazione IRCCS Istituto Neurologico \\ 'Carlo Besta', Milan, Italy
}

\section{Key Words}

Autosomal recessive neurodegenerative disorder $\cdot$ Friedreich ataxia $\cdot$ Laryngeal dystonia

\begin{abstract}
Friedreich ataxia (FRDA) is an autosomal recessive neurodegenerative disorder characterized by progressive gait and limb ataxia, cerebellar, pyramidal and dorsal column involvement, visual defects, scoliosis, pes cavus and cardiomyopathy. It is caused by a homozygous guanine-adenine-adenine (GAA) trinucleotide repeat expansion in intron 1 of the frataxin gene (FXN) on chromosome 9q13-q21.1. Onset is usually in the first or second decade of life; however, late-onset cases of Freidreich ataxia (LOFA), after the age of 25 years, and very lateonset cases of Freidreich ataxia (VLOFA), after the age of 40 years, have been reported. VLOFA is quite rare and usually presents a milder progression of the disease. We report the case of a 64-year-old woman affected with VLOFA whose first symptoms (balance and gait disturbances) occurred at the age of 44 years. At the age of 62 years, she started complaining of a slowly progressive dysphonia showing the clinical aspects of laryngeal dystonia. Molecular analysis showed a 210- and 230-trinucleotide GAA repeat expansion in the two alleles of the FXN gene. Laryngeal dystonia has been reported only in very few cases of ataxia syndrome and never before in FRDA patients. It may represent a rare clinical manifestation of VLOFA thus confirming the high variability of the clinical spectrum of FRDA.
\end{abstract}

(C) 2014 S. Karger AG, Basel 
Rota et al.: Very Late-Onset Friedreich Ataxia with Laryngeal Dystonia

\section{Introduction}

Friedreich ataxia (FRDA) is an inherited autosomal recessive disease usually linked to a homozygous guanine-adenine-adenine (GAA) trinucleotide repeat expansion in the first intron of the frataxin gene (FXN) on chromosome 9q13-q21.1. Only 2-4\% of patients are compound heterozygotes for the GAA expansion on one allele and a deletion or point mutation on the other. There is a direct correlation between the size of the GAA expansion and the phenotype: large GAA repeats are associated with earlier age of onset and a more rapid progression of the disease [1]. The effect of mutation is a reduced synthesis of frataxin, a nuclear encoded mitochondrial protein that plays a role in iron homeostasis [1].

Although FRDA is typically a clinically homogeneous disease that affects young individuals with onset around puberty, some rare cases of late-onset (LOFA) and very late-onset Freidreich ataxia (VLOFA) have been reported as well as heterogeneous clinical presentations [2-4]. The variability in the age of onset and the presence of 'atypical' clinical features can make it difficult to rapidly achieve a correct diagnosis.

We herein expand the disease phenotype by reporting the case of a woman with VLOFA associated with laryngeal dystonia.

\section{Case Report}

A 64-year-old woman complained of slowly progressive imbalance in standing and gait and lower limb stiffness since the age of 44 years. Her symptoms progressed slowly over the years, and after 20 years she had lost most of her autonomy in the basic activities of daily living. At the age of 62 years, she started complaining of a slowly progressive dysphonia, resulting in a whispering voice. ENT examination diagnosed an abductor-type laryngeal dystonia which had no manifest causes, and which was not responsive to L-Dopa, botulinum toxin, and logotherapy.

The patient's past medical history revealed erosive Helicobacter pylori-positive gastritis, irritable bowel syndrome, migraine without aura and cervical and lumbar arthrosis. Her family history was unremarkable. At the age of 64 years, neurological examination showed a spastic-ataxic gait worsened by eye closing, dysarthria with slurred speech, mild limb dysmetria, decreased deep tendon reflexes, extensor plantar responses and loss of vibratory and proprioceptive sensation.

MRI of the brain (fig. 1), MRI spectroscopy and SPECT were unremarkable. Evoked motor and sensory potentials showed slowed central conduction velocity. Nerve conduction studies and electromyography were normal. The videofluoroscopic swallowing study was normal.

Echocardiography showed normal cardiac function with normal thickness of the septal and left ventricular walls. Pulmonary dynamic functional indices and lung volumes were within normal limits, while respiratory muscle strength showed a marked reduction both in inspiration and expiration, indicating involvement of both the diaphragm and skeletal respiratory muscles. Antigangliosides, anti-MAG and onconeural antibodies were negative. Genetic analyses for spinocerebellar ataxias types 1, 2, 3, 6, 15/16, 17 and dystonia type 4 were negative. Molecular analysis showed a 210- and 230-trinucleotide GAA repeat expansion in the two alleles of the $F X N$ gene. 
Rota et al.: Very Late-Onset Friedreich Ataxia with Laryngeal Dystonia

\section{Discussion}

FRDA is usually a disease starting at young age, causing progressive disability and confinement to a wheelchair after 10-15 years from onset. Diagnostic clinical criteria include: (1) age of onset before the end of puberty, (2) progressive ataxia of gait and limbs, (3) absent knee and ankle jerks, (4) dysarthria, (5) pyramidal weakness in lower limbs, (6) axonal damage on electroneurography, and (7) large fiber sensory loss on pathological examination [5]. Hypertrophic cardiomyopathy, increased risk of diabetes mellitus and skeletal abnormalities (i.e., kyphoscoliosis and pes cavus) are also common features [5].

The identification of the disease gene has allowed large screenings in ataxic patients and has resulted in a widening of the spectrum of clinical presentations. About $25 \%$ of patients harboring the FXN molecular defect do not fulfill the above-mentioned clinical criteria and may show 'atypical' features including retained tendon reflexes, tabetic phenotype, unusually slow progression and delayed age of onset [4].

LOFA and VLOFA are defined by onset after the age of 25 and 40 years, respectively [2, 3]. Usually, late-onset forms of FRDA are associated with a milder disease with slower progression and a smaller number of GAA repeats ( $<500$ GAA). VLOFA is very unusual and is caused by $<300$ GAA repeats in at least one of the expanded alleles. It is likely that other genetic and environmental factors as well as levels of residual frataxin in affected tissues may modulate the clinical features, severity and rate of progression of FRDA [1, 3].

Our case is one of the rare cases of VLOFA having $<300$ GAA repeats in both alleles, and represents the first description of abductor laryngeal dystonia in FRDA. Laryngeal dystonia, also known as spasmodic dysphonia, is a focal action-induced form of dystonia that affects the vocal cords and is characterized by involuntary movements of one or more muscles of the larynx during speech [6]. It can be a primary disease (sporadic or idiopathic) or a secondary condition (trauma, infections, drugs, underlying neuromuscular disorders). The most frequent adductor type causes a tight, strangled-sounding voice, often with abrupt starting and stopping. The abductor type causes a breathy, whispering voice that sometimes progresses to aphonia. A mixed-type and an adductor laryngeal breathing dystonia causing persistent inspiratory stridor and paroxysmal cough usually with normal voice have also been reported [6].

Laryngeal dystonia has already been described in only a few cases of SCA20, familial dystonia and multiple system atrophy [7-9]. In some other ataxia syndromes (i.e., SCA1, SCA3), a vocal cord abductor paralysis has been reported [10].

The exclusion of acquired conditions and the progressive course of dysphonia in our patient suggest that laryngeal dystonia may represent a rare clinical manifestation of FRDA. Our report confirms the high variability of the clinical spectrum in FRDA. Presence of laryngeal dystonia in a patient with an ataxic syndrome should lead physicians to consider FRDA in the differential diagnosis.

\section{References}

1 Pandolfo M: Friedreich ataxia: the clinical picture. J Neurol 2009;256:3-8.

2 De Michele G, et al: Late onset Friedreich's disease: clinical features and mapping of mutation to the FRDA locus. J Neurol Neurosurg Psychiatry 1994;57:977-979.

-3 Bidichandani SI, et al: Very late-onset Friedreich ataxia despite large GAA triplet repeat expansions. Arch Neurol 2000;57:246-251.

4 Lynch DR, et al: Friedreich ataxia: effects of genetic understanding on clinical evaluation and therapy. Arch Neurol 2002;59:743-747.

5 Filla A, et al: Accuracy of clinical diagnostic criteria for Friedreich's ataxia. Mov Disord 2000;15:1255-1258. 
Rota et al.: Very Late-Onset Friedreich Ataxia with Laryngeal Dystonia

6 Grillone GA, Chan T: Laryngeal dystonia. Otolaryngol Clin North Am 2006;39:87-100.

7 Storey E, Gardner RJ: Spinocerebellar ataxia type 20. Handb Clin Neurol 2012;103:567-573.

8 Hagenah J, et al: Predominant dystonia with marked cerebellar atrophy: a rare phenotype in familial dystonia. Neurology 2007;68:2157.

9 Merlo IM, et al: Not paralysis, but dystonia causes stridor in multiple system atrophy. Neurology 2002;58:649-652.

10 Shiojiri T, et al: Vocal cord abductor paralysis in spinocerebellar ataxia type 1. J Neurol Neurosurg Psychiatry 1999;67:695.
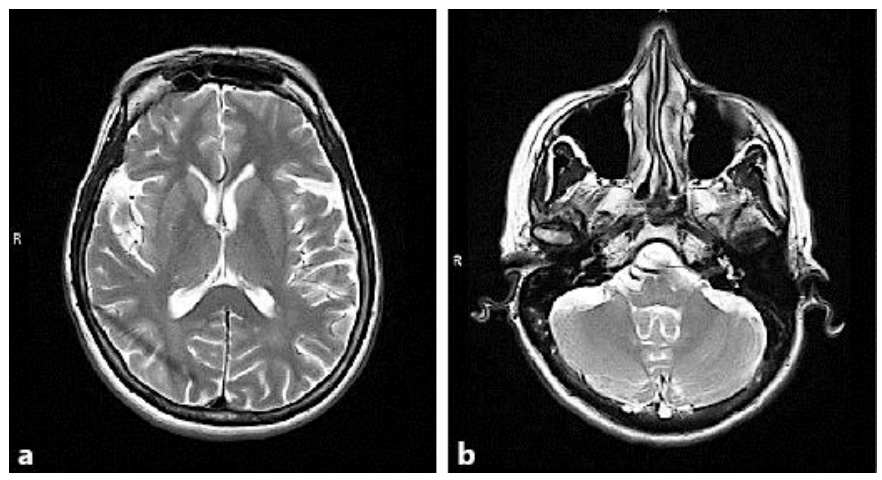

Fig. 1. MRI of the brain. No significant cortical (a) or cerebellar atrophy (b) is present. The brain stem is normal. 